\title{
An Investigation into the Correlation of Small Punch and Uniaxial Creep Data for Waspaloy
}

\author{
T. WILLIAMS, M. EVANS, and W. HARRISON
}

\begin{abstract}
Within the aerospace sector, the understanding and prediction of creep strains for materials used in high-temperature applications, such as Nickel-based super alloys, is imperative. Small punch testing offers the potential for understanding creep behavior using much less material than conventional uniaxial testing but in contrast to uniaxial creep tests, the stress in small punch creep (SPC) tests is multiaxial. SPC testing can be a valuable tool for validating models of creep deformation, but the key to unlocking its full capability is through the accurate correlation of the creep material properties measured through both techniques. As such, the focus of this paper is to correlate the creep behavior of Waspaloy obtained through conventional uniaxial testing to that obtained via small punch creep testing. Recently, and for low chrome steels, this has been achieved through use of the $k_{\mathrm{sp}}$ method, but there are good reasons for believing this technique will not work so well for Nickel-based super alloys. This paper shows this to be the case for Waspaloy and proposes some alternative methods of correlation based on combining the Monkman-Grant relation and the Wilshire equations for both uniaxial and small punch creep. It was found that this latter approach enabled the accurate conversion of SPC minimum displacement rates to equivalent uniaxial minimum creep rates which, when combined with the Wilshire equations, enabled SPC test loads to be converted into equivalent uniaxial stresses (and visa versa) with levels of accuracy that were significantly reduced when compared to using the $k_{\text {sp }}$ method. Further, the random error associated with these conversions were dramatically increased.
\end{abstract}

https://doi.org/10.1007/s11661-021-06318-1

(C) The Author(s) 2021

\section{INTRODUCTION}

THE small punch creep method was originally developed in the 1980s with the idea of estimating the properties of irradiated materials in the power generation industry ${ }^{[1]}$ However, further potential was seen in small scale testing to analyze the residual life of key "in-service" components approaching the end of their life. The traditional uniaxial creep test used to obtain mechanical data is often insufficient due to the large volumes of material that are required to produce test specimens, as these large volumes will in time undermine the integrity of these components in use. ${ }^{[2]}$ However, small punch testing allows for a mini-invasive assessment of the component requiring very little material and therefore less damage is incurred to the component in use. ${ }^{[2]}$ In the small punch creep test, the disc specimens

T. WILLIAMS is with the Institute of Structural Materials, Swansea University, Swansea, SA1 8EN, UK. M. EVANS and W. HARRISON are with the College of Engineering, Swansea University, Swansea, SA1 8EN, UK. Contact e-mail: m.evans@swansea.ac.uk Manuscript submitted January 7, 2021; accepted April 30, 2021.

Article published online May 25, 2021 typically have dimensions of just 8 to $9.5 \mathrm{~mm}$ for the diameter and $500 \mu \mathrm{m}$ for the thickness.

When it comes to the application of the small punch creep test, there have been many reservations surrounding the repeatability and application of the small punch data itself. Reliability and repeatability of testing has been a strong focus within the research community, leading to the establishment of a European Code of Practice. ${ }^{[3]}$ Results from small punch creep (SPC) tests are typically shown through time/displacement curves, which while appearing comparable to that of conventional uniaxial creep curves, in reality are quite different due to the differing creep mechanisms present in each of these test techniques. This is because uniaxial test specimens are only subjected to a single tensile stress state, while in the SPC test the specimen experiences a multiaxial stress state. Therefore, data obtained from SPC tests cannot be used to find or to compare to the values of conventional creep parameters (such as stress)-hence the need for a form of correlation to bridge the gap between the two test types. The $k_{\mathrm{sp}}$ method is based on a constant force-to-stress ratio $(\psi=$ $F / \sigma)$ and is based on the Chakrabarty membrane stretching equations. ${ }^{[4]}$ In this model, $\Psi$ is a function of the test geometry of the small punch creep test and 
the $k_{\mathrm{sp}}$ correction factor. This model, also referred to as the Classic CEN Workshop Agreement model (CWA), has already been applied to a range of materials, where, for example, the work done by Jeffs ${ }^{[5]}$ on CMSX-4 and the work done by Milička and Dobeš on $\mathrm{P} 91^{[6]}$ have met with some success. In some studies on P91 this method has indicated a difference in low and high load conditions, indicating a load dependence. ${ }^{[2]}$ Also, the accuracy of the $k_{\mathrm{sp}}$ method has been shown to be limited to ductile materials - with the work done by Lancaster ${ }^{[7]}$ et al. showing that $k_{\mathrm{sp}}$ correlations for inherently brittle superalloys used for high-temperature applications are not as useful as in the case of Titanium aluminides. Also, it has been indicated that for specific materials the $k_{\mathrm{sp}}$ factor is not a constant, but instead needs to be optimized against uniaxial creep date. ${ }^{[2]}$

Holmstrom et al ${ }^{[8]}$ have proposed some modifications to the CWA model to deal with these limitations. These have involved making $\psi$ dependant upon the amount of displacement present at the minimum displacement rate. One version of this model (the Empirical Force to Stress conversion or the EFS model) has $\Psi$ modeled as a power law function of displacement present at the minimum displacement rate, while another (the Modified CHakrabarty or $\mathrm{MCH}$ model) has $\Psi$ as a linear function of displacement present at the minimum displacement rate. These authors found that EFS and $\mathrm{MCH}$ models were superior to the classical CWA model when tested on P92, F92, and 316L steels. But they also concluded that additional modeling is required when converting SPC minimum displacement rates to uniaxial minimum creep rates.

A number of numerical models have also been proposed by researchers to interpret the stress and temperature relationships between both SPC and uniaxial data. For example, Wang and Evans ${ }^{[9]}$ made use of numerical models of the small punch test together with the Monkman-Grant relation to correlate uniaxial and small punch test data for $0.5 \mathrm{Cr}-0.5 \mathrm{Mo}-0.25 \mathrm{~V}$ and $1.25 \mathrm{Cr}-1 \mathrm{Mo}$ steels. The aim of this paper will be to make use of the Monkman-Grant ${ }^{[10,11]}$ relation for small punch and uniaxial data to convert SPC minimum displacement rates to uniaxial minimum creep rates in the way suggested by Holmstrom et al. ${ }^{[8]}$ and then to incorporate this into a Wilshire type model ${ }^{[12]}$ for uniaxial and small punch data. Then there is no requirement to define and measure $\Psi$. The adequacy of this approach is then compared to the $k_{\mathrm{sp}}$ method using Waspaloy as the test material.

\section{MATERIAL PROPERTIES AND CHARACTERIZATION}

\section{A. Material for Uniaxial Testing}

Thirty-one cylindrical test pieces were machined from an received Waspaloy bar, with a gauge length of $28 \mathrm{~mm}$ and a diameter of $5 \mathrm{~mm}$. The chemical composition of this batch of Waspaloy (in wt. pet) was 19:1Cr; 13:5Co; 0:03C; 0:10Mn; 0:10Si; 0:79Fe; 4:08Mo; 3:15Ti; 1:30Al; 0:005B; 0:07Zr; 0:0025S; 0:01P; 0:10Cu; 5 ppm Ag; 10 ppm $\mathrm{Pb} ; 0: 5 \mathrm{ppm} \mathrm{Bi}$; bal Ni. The material was heat treated for $4 \mathrm{~h}$ at $1353 \mathrm{~K}$ (water quenched), 4 hours at $1123 \mathrm{~K}$ (air cooled), and 16 hours at $1033 \mathrm{~K}$ (air cooled). This resulted in a uniform equiaxed structure of average grain diameter $45 \mu \mathrm{m}$. The microstructure contained uniform $\gamma^{\prime}$ particles of mean diameter $0.3 \mu \mathrm{m}$. The tensile strength $\left(\sigma_{\mathrm{TS}}\right)$ values for this batch of material were $1154,1120,975$, and $827 \mathrm{MPa}$ at $873,923,973$, and $1023 \mathrm{~K}$, respectively.

The specimens were tested in tension over a range of stresses at $873 \mathrm{~K}, 923 \mathrm{~K}, 973 \mathrm{~K}$, and $1023 \mathrm{~K}$ using high precision in Andrade-Chalmers constant-stress machines. ${ }^{[13]}$ Loads and stresses could be applied and maintained to an accuracy of 0.5 pct. In all cases, temperatures were controlled along the gauge lengths and with respect to time to better than $\pm 1 \mathrm{~K}$. The extensometer was capable of measuring tensile strain to better than $10^{-5}$. Loading machines, extensometers, and thermocouples were all calibrated with respect to NPL traceable standards. At $873 \mathrm{~K}$, eight specimens were placed on test over the stress range 1150 to $700 \mathrm{MPa}$, at $923 \mathrm{~K}$ seven specimens were placed on test over the stress range 1000 to $550 \mathrm{MPa}$, at $973 \mathrm{~K}$ nine specimens were placed on test over the stress range 950 to 200 $\mathrm{MPa}$, and at $1023 \mathrm{~K}$ seven specimens were tested over the stress range 700 to $250 \mathrm{MPa}$. Up to 400 creep strain/time readings were taken during each of these tests. Because Waspaloy can serve at temperatures up to $920 \mathrm{~K}$ for critical applications and $1040 \mathrm{~K}$ for less demanding situations, the test program covered stress ranges giving creep lives up to 5500 hours (around $19,852,000$ seconds) at $873 \mathrm{~K}$ to $1023 \mathrm{~K}$. This data set has been published by Wilshire and Scharning ${ }^{[14]}$ and Evans. ${ }^{[15]}$

In all cases, the curves showed conventional primary and tertiary stages. Figure 1(a) shows a typical creep curve recorded at the lowest temperature $(873 \mathrm{~K})$ and Figure 1(b) shows a typical creep curve at the highest test temperature $(1023 \mathrm{~K})$. In both cases, the stress was a mid-value within the test matrix. The strain rates are based on the slope of the measured creep curve using a numerical higher order differentiation method. It can be seen from this figure - by looking either at the creep curve or the strain rate - that the lower temperatures are associated with a more prolonged and pronounced tertiary stage of creep. At $1023 \mathrm{~K}$, the minimum creep rate occurs very early on in the test, with noticeable necking present towards the end of the test. At $873 \mathrm{~K}$, the minimum creep rate occurs towards the middle of the test, with necking again visible at the end of the creep curve. But all failed specimens showed evidence of grain boundary cavitation. ${ }^{15]}$

\section{B. Material for Small Punch Testing}

Discs with a radius of $8 \mathrm{~mm}$ and a thickness of 1000 $\mu \mathrm{m}$ were cut from an as-received Waspaloy bar, with chemical composition (in wt. pet) $19: 5 \mathrm{Cr} ; 13: 5 \mathrm{Co}$; 0:08C; 4:3Mo; 3:0Ti; 1:30Al; 0:006B; balance Fe. This supplied material is therefore very similar to that used for the uniaxial test specimens. The cut discs were ground down using progressively finer grit papers until a 
$500 \pm 5 \mu \mathrm{m}$ thickness was achieved in accordance with the SPC Euro Co ${ }^{[16]}$ The as-received bar had a typical heat treatment of $1268 \mathrm{~K}$ to $1313 \mathrm{~K}$ for 4 hours, air cooled, followed by a stabilization period at $1118 \mathrm{~K}$ for 4 hours, air cooled, and aged at $1033 \mathrm{~K}$ for 16 hours, air cooled. The grain size ranged between 16 and $127 \mu \mathrm{m}$. The heat treatment experience by this material is therefore very similar to that experienced by the material used for uniaxial test specimens. The grains sizes were also similar. Figure 2(a) shows, through an SEM image of this as-received Waspaloy bar, that the microstructure consists of primary $\gamma^{\prime}$ precipitates evenly distributed within a $\gamma$, with finer secondary $\gamma^{\prime}$ precipitates formed during heat treatment after the aging stage. From the BSE scans in Figure 2(b) it is clear that dark inclusions present in the coarse grain matrix of Figure 2(a) are TiN. These inclusions, alongside $\mathrm{NiC}$, can form in Waspaloy during primary processing of superalloys. ${ }^{[1]}$

For polycrystalline Nickel-based superalloys used at elevated temperatures, grain boundaries are used to modify material properties such as fatigue, creep, and corrosion resistance. This is completed through the addition of coincident site lattices (CSL). The degree of misorientation and thus coherency of a grain boundary can be defined as the CSL and each CSL has a subsequent $\Sigma$ value. Low CSL boundaries $(\Sigma>29)$ contain large defects and vacancies and are therefore less desirable as they promote creep diffusion mechanisms. However, high CSL boundaries $(\Sigma<29)$ are coherent and contain less crystal defects, making them creep resistant at these elevated temperatures. A common example of a high CSL boundary is twins which occur at a misorientation of $60 \mathrm{deg} \pm 5$ giving them a $\Sigma$ value of 3. The EBSD data summarized in Figures 2(c) and (d) show the grain boundary types of the as-received Waspaloy used for SPC testing in this paper. While some low CSL boundaries can be observed (Blue and Pink), a large proportion of twinning $(\Sigma=3)$ is also observed. This leads to the superior creep properties at elevated temperature conditions for this material.

The discs were tested on a dead-weight test frame assembly, in which a load pan applied a load through a central axis to the circumferential clamped discs, over a range of loads at $923 \mathrm{~K}, 973 \mathrm{~K}$, and $1023 \mathrm{~K}$. The maximum force at failure $\left(F_{\max }\right)$, obtained in a SPC test where the force is controlled to maintain a constant displacement rate until failure, were 1565, 1520, and $1450 \mathrm{~N}$ at $923 \mathrm{~K}, 973 \mathrm{~K}$, and $1023 \mathrm{~K}$, respectively. At $923 \mathrm{~K}$, three discs were tested at loads of $700 \mathrm{~N}, 650 \mathrm{~N}$, and $600 \mathrm{~N}$, at $973 \mathrm{~K}$, three discs were tested at loads of $600 \mathrm{~N}, 550 \mathrm{~N}$, and $500 \mathrm{~N}$ and at $1023 \mathrm{~K}$ three discs were tested at loads of $500 \mathrm{~N}, 450 \mathrm{~N}$, and $400 \mathrm{~N}$. At the lowest temperature the test was repeated three times at each of the stated loads, and all analyses were based on the average recorded failure time and minimum displacement rates. A repeat test was also carried out at 973 $\mathrm{K}-600 \mathrm{~N}$. These temperature and load conditions gave creep lives of between 1015 hours down and 6 hours. Displacement/time readings were taken throughout the tests from two linear variable displacement transducers, one positioned near the load pan to take displacement readings from the topside of the disc and the other placed in the lower die to record displacement of the underside of the disc.

Normal displacement curves were observed for each specimen. In Figures 3(a) and (c) displacement and

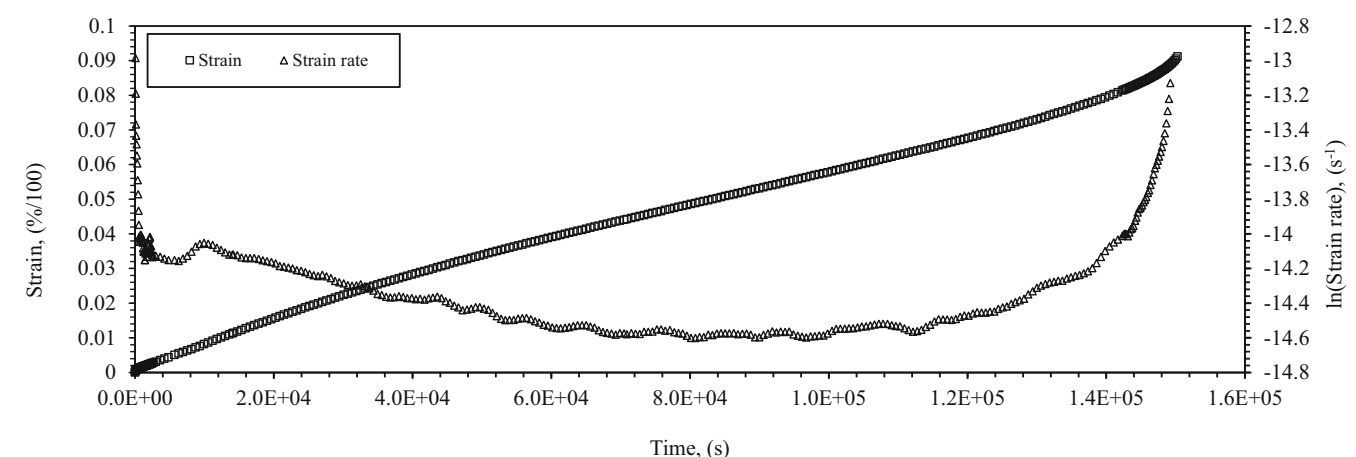

(a)

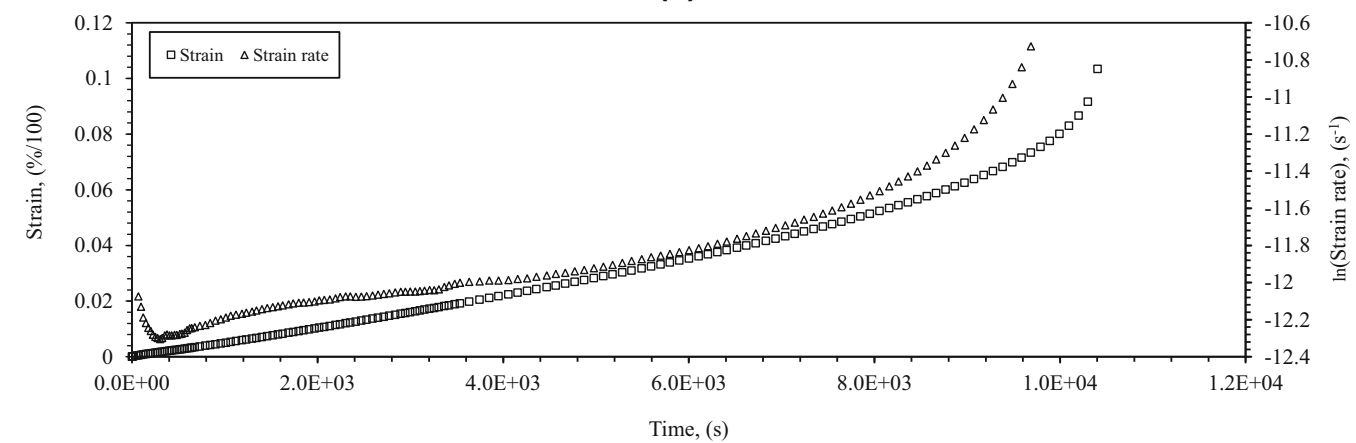

(b)

Fig. 1 - Creep strain and creep rates recorded at (a) 873 K-900 MPa and (b) 1023 K-600 MPa. 


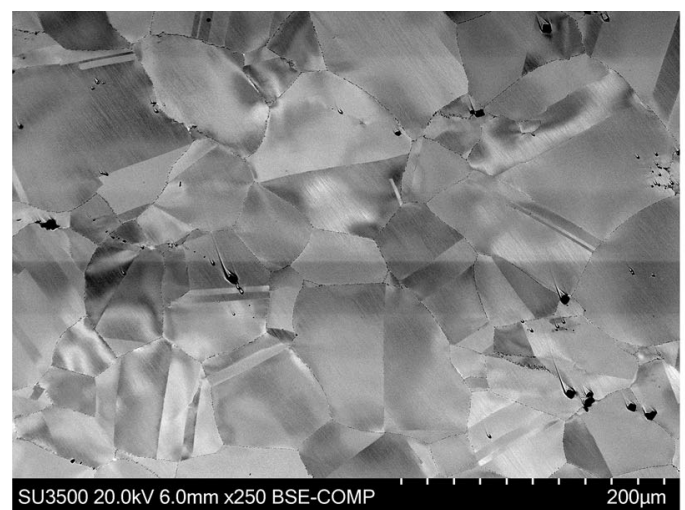

(a)

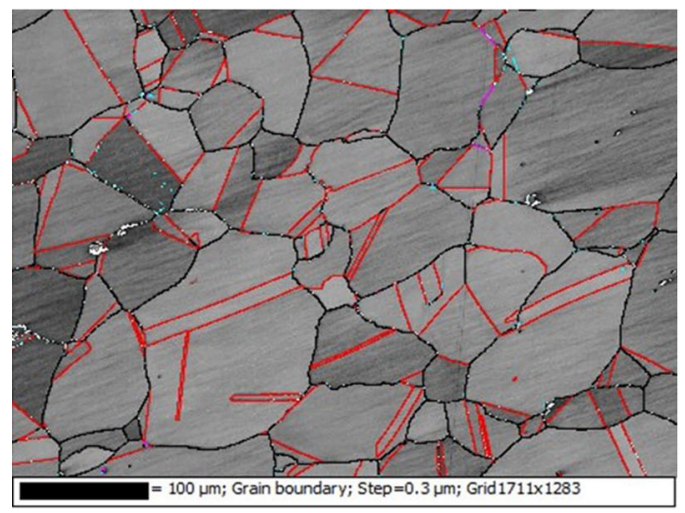

(c)

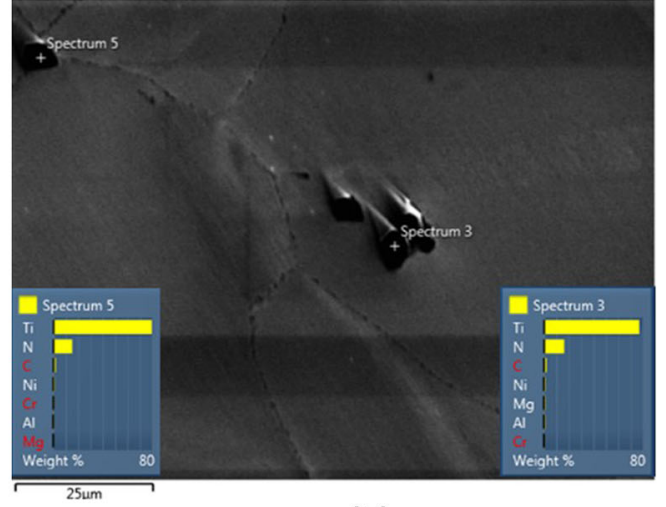

(b)

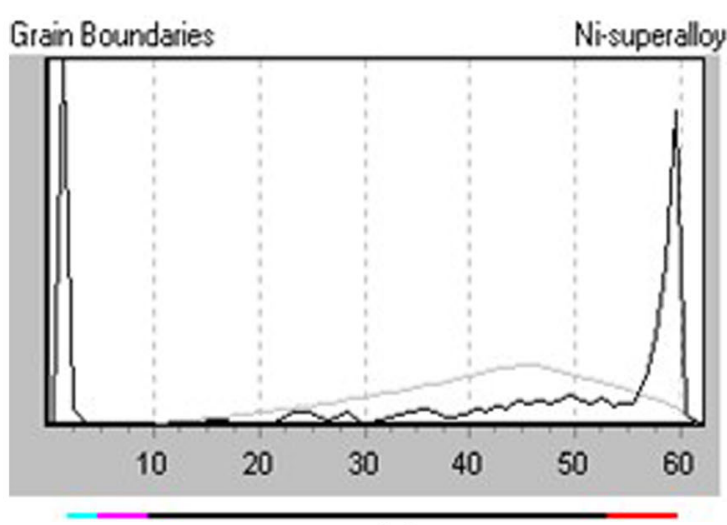

(d)

Fig. 2-Images of the as-received Waspaloy material used for SPC testing: (a) An SEM microstructure image, (b) a subsequent BSE scan, (c) an EBSD image of the grain boundary types, and $(d)$ computed $\Sigma$ values.

displacement rate curves are shown for specimens tested at the highest and lowest temperatures. The test curves showed no sign of premature cracking, which would have revealed itself in multiple points of minima for the log displacement rates. Again the effect of increasing temperature appears to be in shortening the period of time where decelerating displacement is occurring and lengthening the time over which the log displacement rate is approximately constant. Figures $3(\mathrm{~b})$ and (d) below contain Keyence images of one of the SPC discs (tested at $923 \mathrm{~K}$ and $650 \mathrm{~N}$ ) post creep rupture. Figure 3(b) shows a micrograph of the discs fracture surface and Figure 3(d) a cross section of the ruptured disc. As expected of high-temperature creep tests, Figure 3(b) shows evidence of brittle failure as it is clear that the cracks on the underside of the disc grew in a radial fashion from the center of the disc finally resulting in an outward rupture. This is due to a lack of elasticity upon creep stain accumulation, thus causing the bottom of the disc to be in tension. Further, the fracture surface itself has a dimpled look, which is typical of intergranular cleavage, both characteristics observed in inherently brittle materials such as $\gamma \mathrm{TiAl}$ and $\mathrm{Mg}$ Alloys. The failure mechanism was confirmed by Scanning Electron Microscope images of the ruptured discs cross section as shown in Figure 3(e). Both wedge cracking and the formation of voids (circled) can be observed along the grain boundaries in the failed sample exposed to creep at $923 \mathrm{~K}$ at a load of $650 \mathrm{~N}$.

\section{METHODOLOGIES}

\section{A. Some Established SPC Conversions Models}

The current focus in the field of small punch creep testing is gaining an understanding of the relationship between the small punch force and uniaxial creep stress. The key to this relationship is a form of conversion that enables the small punch force to be represented as a equivalent uniaxial stress. The most common technique for the empirical correlation of SPC and uniaxial creep data is through the creep correlation factor, $k_{\mathrm{sp}}$. The $k_{\mathrm{sp}}$ factor was first introduced in 2006 when the European standard released the "Europe Code of Practice for Small Punch Creep Testing." 16$]$ This method uses an equation derived from the Chakrabarty membrane stretching theory whereby the ratio of the small punch force $\mathrm{F}$ to the uniaxial stress, $\sigma$, is dependent on the disc and testing geometries. The equation has the form

$$
\frac{F}{\sigma}=\psi_{\mathrm{CWA}}=3.332 k_{\mathrm{sp}} R^{-0.202} r^{1.192} h_{0},
$$

where $R$ is the die hole radius, $r$ is the punch head radius, $h_{0}$ is the thickness of the specimen, and $k_{\mathrm{sp}}$ a 


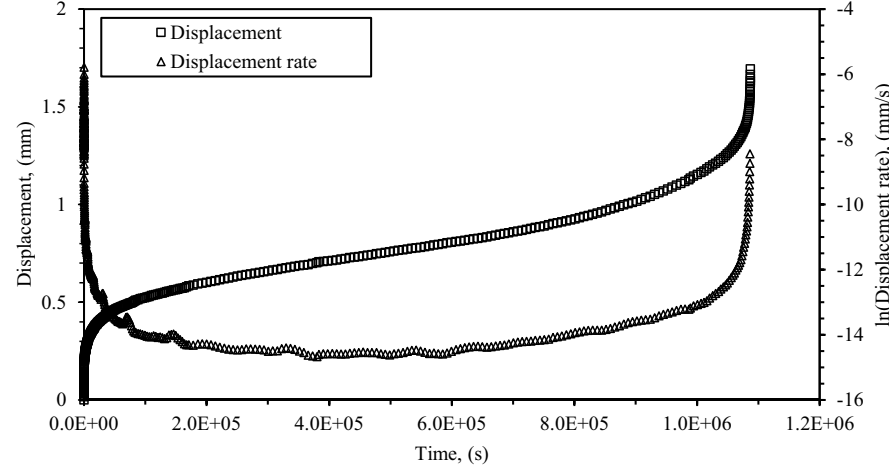

(a)

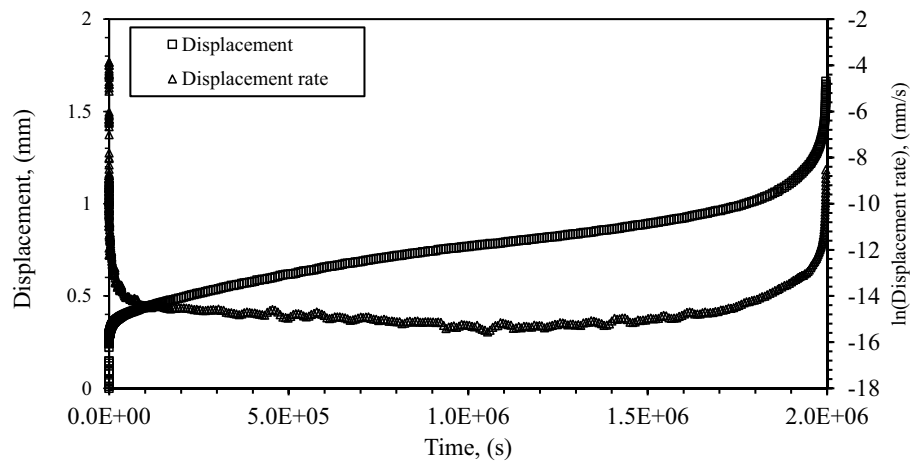

(c)

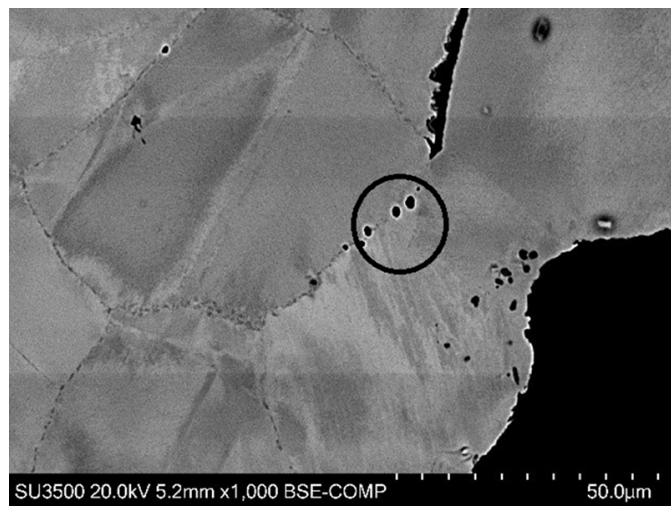

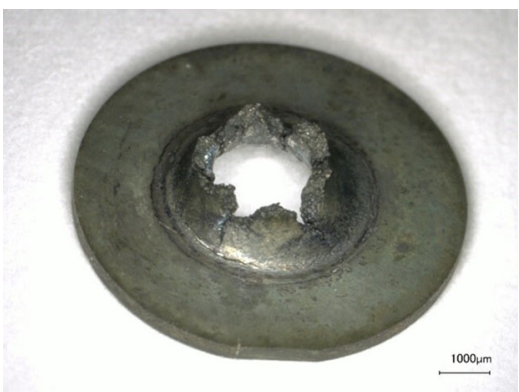

(b)

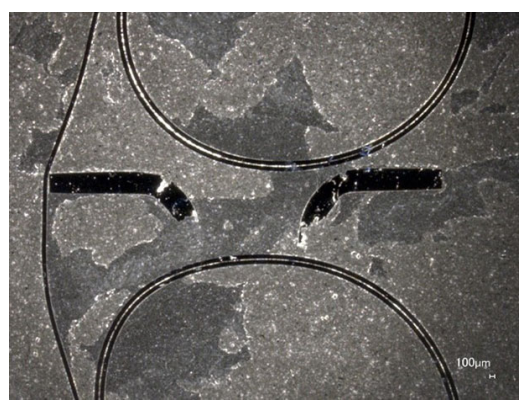

(d)

(e)

Fig. 3-Displacement and displacement rates recorded at (a) $923 \mathrm{~K}-650 \mathrm{~N},(b)$ micrograph of the failed discs fracture surface (as tested as 923 $\mathrm{K}-650 \mathrm{~N}),(c)$ displacements recorded at $1023 \mathrm{~K}-450 \mathrm{~N},(d)$ a cross section of the ruptured disc, and (e) scanning electron microscope images of the ruptured discs cross section.

material-specific ductility parameter. It is often referred to as a correlation factor because its value is adjusted so as to fit with the uniaxial date on materials with varying degrees of ductility. After the release of the CEN SPCT code of practice, ${ }^{[16]}$ the most commonly used test apparatus geometry in Europe use values of $R=2 \mathrm{~mm}, r=1.25 \mathrm{~mm}$, and $h_{0}=0.5 \mathrm{~mm}$ and thus Eq. [1a] reduces to

$$
\frac{F}{\sigma}=1.8897 k_{\text {sp }}
$$

In this approach, the equivalent stress associated with a SPC test force is therefore given by

$$
\sigma=0.5292 \frac{F}{k_{\mathrm{sp}}} .
$$

This $k_{\mathrm{sp}}$ approach is quite restrictive in nature, and this can be most easily illustrated by assuming that a power (or Norton's) law model describes adequately the relationships between i. minimum uniaxial creep rates 
$\dot{\varepsilon}_{\min }$ and uniaxial stress $\sigma$ and ii. minimum displacement rates $\dot{u}_{\min }$ and the SPC force $F$

$$
\begin{gathered}
\dot{\varepsilon}_{\min }=A \sigma^{n} \\
\dot{u}_{\min }=B(F)^{m},
\end{gathered}
$$

where $A, B, n$, and $m$ are model parameters. Substituting Eq. [1c] into Eq. [2a] enables uniaxial creep data to be converted into equivalent SPC data

$$
\dot{u}_{\min }=A\left[0.5292 \frac{F}{k_{\mathrm{sp}}}\right]^{n}=A\left[\frac{0.5292}{k_{\mathrm{sp}}}\right]^{n}(F)^{n} .
$$

In the $k_{\mathrm{sp}}$ method, no distinction is made between minimum creep rates and minimum displacement rates - they are taken to be equivalent. This enables $\dot{\varepsilon}_{\text {min }}$ to be replaced by $\dot{u}_{\text {min }}$ on the left-hand side of Eq. [2c]. A comparison of Eqs. [2b] and [2c] then makes clear the fact that the $k_{\mathrm{sp}}$ method requires the restriction that $m=n$ and $B=A\left[0.5292 / k_{\mathrm{sp}}\right]^{n}$ to hold true.

Further, the $k_{\mathrm{sp}}$ method makes no adjustment for measured minimum creep or displacement rates when converting stresses (forces) to forces (stresses). This however is taken into account in the EFS and $\mathrm{MCH}$ models. In the ESP model

$$
\frac{F}{\sigma}=\psi_{\mathrm{EFS}}=\alpha_{0}\left(u_{\mathrm{min}}\right)^{\alpha_{1}}
$$

where $u_{\min }$ is the displacement ( $\mathrm{mm}$ ) that occurs when the displacement rate, $\dot{u}$ is at a minimum. In the $\mathrm{MCH}$ model

$$
\frac{F}{\sigma}=\psi_{\mathrm{MCH}}=\alpha_{2}+\alpha_{3} u_{\min }
$$

$\alpha_{0}$ to $\alpha_{3}$ are parameters that need to be estimated based on maximizing the correlation between the small punch and uniaxial data available on a specific material. To the extent that $u_{\min }$ is likely to vary with force and temperature, it is suggested that in these models $\Psi$ is test condition dependent.

\section{B. An Modified Approach}

As Holmstrom et al. ${ }^{[8]}$ concluded, the use of Monkman-Grant type relations ${ }^{[10,11]}$ are likely to be needed to improve the universal applicability of methods based on $\Psi$. In this section, such relations are combined with a Wilshire model for both uniaxial and SPC test data to eliminate the need for the computation of $\Psi$ in the conversion of a uniaxial stress to an SPC force. The Monkman-Grant relation is a commonly used predictive model which typically relates a uniaxial time to failure, $t_{\mathrm{f}, \mathrm{ua}}$, to the minimum creep rate, $\dot{\varepsilon}_{m}$, with a relationship of the form:

$$
t_{\mathrm{f}, \mathrm{ua}}=a_{1}\left(\dot{\varepsilon}_{\mathrm{min}}\right)^{b_{1}},
$$

where $a_{1}$ and $b_{1}$ are material constants typically estimated through linear regression. They are thought to be material specific, and therefore independent of test conditions. For many materials, especially low chrome steel alloys, $b_{1}$ tend to be around 1 in value. Research on high-temperature alloys, such as $1 \mathrm{Cr}-1 \mathrm{Mo}$ steel, and $2.25 \mathrm{Cr}-1 \mathrm{Mo}$ steel conducted by Song, ${ }^{[17]}$ found the values of $a_{1}$ and $b_{1}$ to be 0.0528 and 1.016 , respectively. While work by Evans ${ }^{[18]}$ on $1 \mathrm{CrMoV}$ steel produced values of 4.775 and 0.967 , respectively.

This relation was initially identified from uniaxial creep data, but work done by Dobes and Milicka ${ }^{[11]}$ applied this same relation to the time to failure from an SPC test, $t_{\mathrm{f}, \mathrm{spc}}$, and the minimum displacement rate, $\dot{u}_{\min }$

$$
t_{\mathrm{f}, \mathrm{spc}}=a_{0}\left(\dot{u}_{\mathrm{min}}\right)^{b_{0}}
$$

These authors concluded that this relationship was just as pronounced as that found for uniaxial data. It is thus possible to adjust the minimum displacement rates at each SPC test failure time to bring them into line with minimum creep rates, i.e., calculate equivalent minimum creep rates. This is done by setting $t_{\mathrm{f}, \mathrm{spc}}=t_{\mathrm{f} \text {,ua }}$ in Eqs. [4a] and [4b]. For many materials $b_{0}=b_{1}=1$ in which case the equivalent minimum creep rate is given by

$$
\ln \left[\dot{\varepsilon}_{\min }\right]=\ln \left[a_{0}\right]-\ln \left[a_{1}\right]+\ln \left[\dot{u}_{\min }\right],
$$

where $\ln$ stands for the natural log. For other materials $b_{0}=b_{1} \neq 1$ in which case

$$
\ln \left[\dot{\varepsilon}_{\min }\right]=\frac{\ln \left[a_{0}\right]-\ln \left[a_{1}\right]}{b_{1}}+\ln \left[\dot{u}_{\min }\right] .
$$

But more generally $b_{0} \neq b_{1}$ in which case (obtained by equating Eqs. [4a] and [4b]

$$
\ln \left[\dot{\varepsilon}_{\min }\right]=\frac{\ln \left[a_{0}\right]-\ln \left[a_{1}\right]}{b_{1}}+\frac{b_{0}}{b_{1}} \ln \left[\dot{u}_{\min }\right] .
$$

Minimum rates being tied together in this way further imply that force and stress are also tied together. Exactly how they are tied together depends on the way in which stress varies with the minimum creep rate and the way in which the minimum displacement rate varies with force. The Wilshire equations ${ }^{[2]}$ are a fairly new approach to model uniaxial creep data that have not only proved to provide very reliable interpolated stresses for a wide variety of high-temperature materials, but also produce very good extrapolations out to very low stresses (and high failure times). Its use in modeling SPC data is not so wide spread. The Wilshire equation for the uniaxial minimum creep rate is a sigmoidal S-shaped curve at a fixed temperature

$$
\frac{\sigma}{\sigma_{\mathrm{TS}}}=\exp \left\{-k_{2 j}\left[\dot{\varepsilon}_{\min } \exp \left(\frac{Q^{*}}{R T}\right)\right]^{v_{j}}\right\}
$$

$j=1$ when $\sigma / \sigma_{\mathrm{TS}} \leq \sigma_{1}^{c} ; j=2$ when $\sigma_{1}^{c}<\sigma / \sigma_{\mathrm{TS}} \leq \sigma_{2}^{c}$; $\ldots ; j=p$ when $\sigma / \sigma_{\mathrm{TS}}>\sigma_{p}^{c}$

$$
\sigma_{1}^{c}<\sigma_{2}^{c}<\cdots<\sigma_{p}^{c}
$$

where $T$ is the absolute temperature, $\sigma_{\mathrm{TS}}$ the tensile strength, $\mathrm{R}$ the universal gas constant. $Q^{*}$ is the activation energy for $\dot{\varepsilon}_{\min }$, while $k_{2 j}$ and $v_{j}$ are further parameters that apply in each of the $p$ normalized 
stress ranges. $\sigma_{j}^{c}$ are critical values for the normalized stress and so fall between 0 and 1 . In this approach, there are $\mathrm{p}$ creep regimes that occur in distinct ranges for the normalized stress and the p versions of Eq. [6] then apply to each regime. Typically, $\mathrm{p}$ varies between 0 and 4 depending on the material being studied. Fortunately, it is relatively straightforward to linearize this model, so that linear least squares can be used to indirectly estimate the unknown parameters $\left(k_{2 j}, v_{j}, Q^{*}\right)$ from the uniaxial date

$$
\ln \left(\dot{\varepsilon}_{\min }\right)=c_{0 j}+c_{1 j} \sigma^{*}+c_{2} \frac{1000}{R T},
$$

where $\sigma^{*}=\ln \left[-\ln \left(\sigma / \sigma_{\mathrm{TS}}\right)\right]$ and $c_{0 \mathrm{j}}$ to $c_{2}$ are related to the parameters $\left(k_{2 j}, v_{j}, Q^{*}\right)$ in each of the $p$ normalized stress ranges (for example $c_{1 j}=1 / v_{j}$ and $c_{2}=Q^{*}$ in $\left.\mathrm{kJ} \mathrm{mol}^{-1}\right)$. The Wilshire equations use in modeling SPC data is not so prolific, but its equivalent takes the form

$$
\ln \left(\dot{u}_{\min }\right)=d_{0 j}+d_{1 j} F^{*}+d_{2} \frac{1000}{R T},
$$

where $F^{*}=\ln \left[-\ln \left(F / F_{\max }\right)\right] . F_{\max }$ is the maximum force at failure obtained in a SP test where the force is controlled to maintain a constant displacement rate until failure.

The Wilshire equations for times to failure recorded from uniaxial and SPC tests, respectively, have the same form as above

$$
\begin{gathered}
\ln \left(t_{\mathrm{f}, \text { ua }}\right)=e_{0 j}+e_{1 j} \sigma^{*}+e_{2} \frac{1000}{R T} \\
\ln \left(t_{\mathrm{f}, \mathrm{spc}}\right)=g_{0 j}+g_{1 j} F^{*}+g_{2} \frac{1000}{R T} .
\end{gathered}
$$

Two conversion approaches now present themselves. The first involves converting a minimum displacement rate measured at a particular force and temperature, into an equivalent minimum creep rate using the most appropriate version of Eq. [5]. The resulting equivalent minimum creep rate is then inserted into Eq. [7a] and Eq. [7b] into the resulting equation. The result, together with a value for $T$, can be used to find the force equivalent to the stress used in the uniaxial creep test $\left(F^{*}\right.$ is easily converted too $F$ using the known maximum force for that temperature). When using Eq. [5c], this gives the conversion equation

$$
\begin{aligned}
F^{*}= & \left\{\frac{b_{1} c_{0 j}}{b_{0} d_{1 j}}-\frac{\ln \left[a_{0}\right]-\ln \left[a_{1}\right]}{b_{0} d_{1 j}}-\frac{d_{0 j}}{d_{1 j}}\right\}+\frac{b_{1} c_{1 j}}{b_{0} d_{1 j}} \sigma^{*} \\
& +\left\{\frac{b_{1} c_{2}}{b_{0} d_{1 j}}-\frac{d_{2}}{d_{1 j}}\right\} \frac{1000}{R T}
\end{aligned}
$$

which can also be re-arranged to find the uniaxial stress equivalent to a particular SPC force. The second involves fewer steps. It simply involves setting Eqs. [8a] and [8b] equal to each other to obtain the conversion equation. For the conversion of uniaxial stress to SPC force this conversion equation takes the form

$$
F^{*}=\frac{\left(e_{0 j}-g_{0 j}\right)}{g_{1 j}}+\frac{e_{1 j}}{g_{1 j}} \sigma^{*}+\frac{\left(e_{2}-g_{2}\right)}{g_{1 j}} \frac{1000}{R T}
$$

which can be re-arranged to find the uniaxial stress equivalent to a particular SPC force.

Equations [9a] and [9b] do not require the use of $\Psi$, but to work, the parameters $a, b, c, d, e$, and $g$ must be estimated from the date collected on uniaxial and SPC tests. This is also true for the other models mentioned above $-k_{\mathrm{sp}}$ needs to be estimated from a comparison of uniaxial to SPC data as its value is materials specific, and in the ESP method data on displacement (and possibly creep rates) are needed.

\section{MEASURING THE ACCURACY OF EACH CORRELATION METHOD}

To assess the accuracy of the above approaches to converting force to stress and visa versa, the Wilshire equations for time to failure can be used to obtain interpolated uniaxial stresses, SPC forces, and times to failure along the lines used by Holmstrom et al. ${ }^{[8]}$ The Wilshire interpolated uniaxial stress $\sigma_{\mathrm{I}}$ corresponding to a measured SPC failure time is calculated from

$$
\sigma^{*}=-\frac{e_{0 j}}{e_{1 j}}-\frac{e_{2}}{e_{1 j}} \frac{1000}{R T}+\frac{1}{e_{1 j}} \ln \left(t_{\mathrm{f}, \mathrm{spc}}\right)
$$

with $\sigma_{\mathrm{I}}=\exp \left(-\exp \left(\sigma^{*}\right)\right) \sigma_{\text {UTS }}$. The Wilshire interpolated force $\mathrm{F}_{\mathrm{I}}$ corresponding to a measured uniaxial failure time is calculated as

$$
F^{*}=-\frac{g_{0 j}}{g_{1 j}}-\frac{g_{2}}{g_{1 j}} \frac{1000}{R T}+\frac{1}{g_{1 j}} \ln \left(t_{\mathrm{f}, \text { ua }}\right)
$$

with $F_{\mathrm{I}}=\exp \left(-\exp \left(F^{*}\right)\right) F_{\max } \cdot \sigma_{\mathrm{I}}$ and $F_{\mathrm{I}}$ can be treated as if they are actual stresses and forces with which to compare to the converted values given by the above approaches.

Alternatively, Eqs. [1] and [9] can be used to calculate the equivalent SPC force to the uniaxial stresses making up the uniaxial test data set described in Section II. This force can then be substituted into Eq. [8b] to work out the predicted SPC failure time corresponding to the uniaxial test conditions. These predicted SPC failure times can then be compared to the actual uniaxial failure times to assess the suitability of Eqs. [1] and [9] in calculating equivalent forces.

In the following equations $y_{i}^{a}$ can refer to either $\sigma_{\mathrm{I}}$ or $F_{\mathrm{I}}$ or the actual uniaxial failure time at test condition $i$. $y_{i}^{p}$ can represent either the equivalent force or stress calculated using Eqs. [1] and [9], or the predicted failure time obtained using Eq. [8b] when using the equivalent force calculated using Eqs. [1] and [9]. Then the mean square error (MSE) is given by

$$
\operatorname{MSE}=\frac{\sum_{i=1}^{k}\left(y_{i}^{a}-y_{i}^{p}\right)^{2}}{k},
$$


where there are $k$ comparisons between $y^{a}$ and $y^{p}$. While the squaring of the prediction error prevents under predictions being offset by over predictions in the averaging procedure, the MSE provides no sense of scale for the prediction errors. One simple modification of Eq. [11a] that introduces a sense of scale is to replace $y^{a}$ and $y^{p}$ with the natural $\log$ of their values. This scaling comes about because when using the natural logs, the MSE associated with the logged data is approximately equal to the mean square percentage error (MSPE) associated with the raw (untransformed) data:

$$
\frac{\sum_{i=1}^{k}\left(\ln \left[y_{i}^{a}\right]-\ln \left[y_{i}^{p}\right]\right)^{2}}{k} \cong \mathrm{MSPE}=\frac{\sum_{i=1}^{k}\left(\left[y_{i}^{a}-y_{i}^{p}\right] / y_{i}^{p}\right)^{2}}{k}
$$

with this approximation being better the smaller are the percentage errors. This MSPE can be decomposed in one of two ways. Theil ${ }^{[19]}$ has shown that

$$
\begin{aligned}
M S P E & =\left(\overline{\ln \left[y^{a}\right]}-\overline{\ln \left[y^{p}\right]}\right)^{2}+\operatorname{Var}\left(\ln \left[y_{i}^{a}\right]-\ln \left[y_{i}^{p}\right]\right) \\
& =\left(\overline{\ln \left[y^{a}\right]}-\overline{\ln \left[y^{p}\right]}\right)^{2}+\left(\sigma^{a}-\sigma^{p}\right)^{2}+2(1-r) \sigma^{a} \sigma^{p},
\end{aligned}
$$

where $\overline{\ln \left[y^{a}\right]}$ is the mean of the variable $\ln \left[y_{i}^{a}\right]$ (called the $\log$ mean), $\overline{\ln \left[y^{b}\right]}$ is the mean of the variable $\ln \left[y_{i}^{p}\right]$, $\sigma^{a}$ is the standard deviation for the variable $\ln \left[y_{i}^{a}\right], \sigma^{p}$ is the standard deviation for the variable $\ln \left[y_{i}^{a}\right]$, and $r$ is the correlation coefficient between $\ln \left[y_{i}^{a}\right]$ and $\ln \left[y_{i}^{p}\right]$. $\operatorname{Var}\left(\ln \left[y_{i}^{a}\right]-\ln \left[y_{i}^{p}\right]\right)$ reads the variance of the percentage prediction error $\left(y_{i}^{a}-y_{i}^{p}\right) / y_{i}^{p}$. Dividing both sides by the MSPE defines what Theil called the proportions of inequality

$$
\begin{aligned}
1 & =\frac{\left(\overline{\ln \left[y^{a}\right]}-\overline{\ln \left[y^{p}\right]}\right)^{2}}{\operatorname{MSPE}}+\frac{\left(\sigma^{a}-\sigma^{p}\right)^{2}}{\operatorname{MSPE}}+\frac{2(1-r) \sigma^{a} \sigma^{p}}{\mathrm{MSPE}} \\
& =U^{M}+U^{S}+U^{C}
\end{aligned}
$$

The bias proportion $U^{\mathrm{M}}$ is an indication of systematic error since it measures the extent to which the average values of the predicted and actual logged series deviate from each other. The variance proportion $U^{\mathrm{S}}$ indicates the ability of the model to replicate the extent to which actual and predicted series deviate from their log mean values as a result of changes in test conditions. Therefore, this is also a systematic error. The covariance proportion $U^{\mathrm{C}}$ measures random or unsystematic error and represents the remaining prediction error after deviations from average values have been accounted for. Experimental errors associated with measuring failure times means that it is unreasonable to expect the predictions made from any model to be perfectly correlated with actual values and so this component is less of a concern than the other two.

\section{RESULTS AND DISCUSSION}

\section{A. Monkman-Grant Relations}

In Figure 4, the uniaxial failure times are plotted against the minimum creep rates and these test data points are shown as solid squares. The MonkmanGrant relation for this uniaxial data is shown as a solid line, and this relation explains nearly 98 pct of the variation in the uniaxial failure times. In relation to Eq. [4a], the parameter $a_{1}=1.2096$ and $b_{1}=-0.778$. The SPC failure times are plotted against the minimum displacement rates and these test data points are shown as open squares. The Monkman-Grant relation for the SPC data is shown as a dashed line, and this relation explains nearly 91 pct of the variation in the SPC failure times. In relation to Eq. [4b] the parameter $a_{0}=104.5386$ (as the units for creep and displacement rates are different) and $b_{0}=-0.556$. With $b_{1} \neq b_{0}$, the most appropriate equation for converting minimum displacement rates into minimum creep rates is Eq. [5c], and the resulting equivalent minimum creep rates are shown as open circles in Figure 4. It can be seen that these data points fall nicely around the uniaxial Monkman-Grant relation.

\section{B. Wilshire Equations}

Figure 5(b) shows a typical display of the Wilshire equation for uniaxial failure times. The horizontal axis shows the temperature-compensated uniaxial failure times using an activation energy of $204 \mathrm{~kJ} \mathrm{~mol}^{-1}$ (or $e_{2}=204 \mathrm{in}$ Eq. [8a]). The vertical axis is a $\log -\log$ transformation of the normalized stress, with the dashed line showing the best fit to the data points. The best fit (i.e., largest $R^{2}$ value) is obtained by allowing for a discontinuity at $\sigma^{*}=-1.45$. This best fit line is given by Eq. [8a] with $e_{0}=-13.875$ and $e_{1}=1.438$ below $\sigma^{*}=-1.45$ and with $e_{0}=-9.606$ and $e_{1}$ $=4.380$ above $\sigma^{*}=-1.45$. The fit has an $R^{2}$ value of nearly 95 pct.

The same display is used to represent the Wilshire equation for uniaxial minimum creep rates in Figure 5(a). The horizontal axis shows the temperature-compensated minimum creep rates using an activation energy of $231 \mathrm{~kJ}$ $\mathrm{mol}^{-1}$ (or $c_{2}=231$ in Eq. [7a]). The different estimated activation energy obtained when using minimum creep rates reflects the fact that $b_{1}$ in the uniaxial MonkmanGrant relation does not equal - 1. Again the dashed line shows the best fit to the data points. The best fit (i.e., largest $R^{2}$ value) is obtained by allowing for a discontinuity at $\sigma^{*}=-1.45$. This best fit line is given by Eq. [7a] with $c_{0}=14.983$ and $c_{1}=-1.411$ below $\sigma^{*}=-1.45$ and with $\mathrm{c}_{0}=8.640$ and $c_{1}=-5.783$ above $\sigma^{*}=-$ 1.45. The fit has an $R^{2}$ value of just over 95 pet. Whittaker et al. ${ }^{[20]}$ interpretation of this break point was related to dislocation interaction with $\gamma^{\prime}$ precipitates below the yield stress. However, significantly increased dislocation densities at stresses above yield results in forest hardening becoming the primary mechanism controlling dislocation movement. Thus break points are attributed to changing creep mechanisms. 


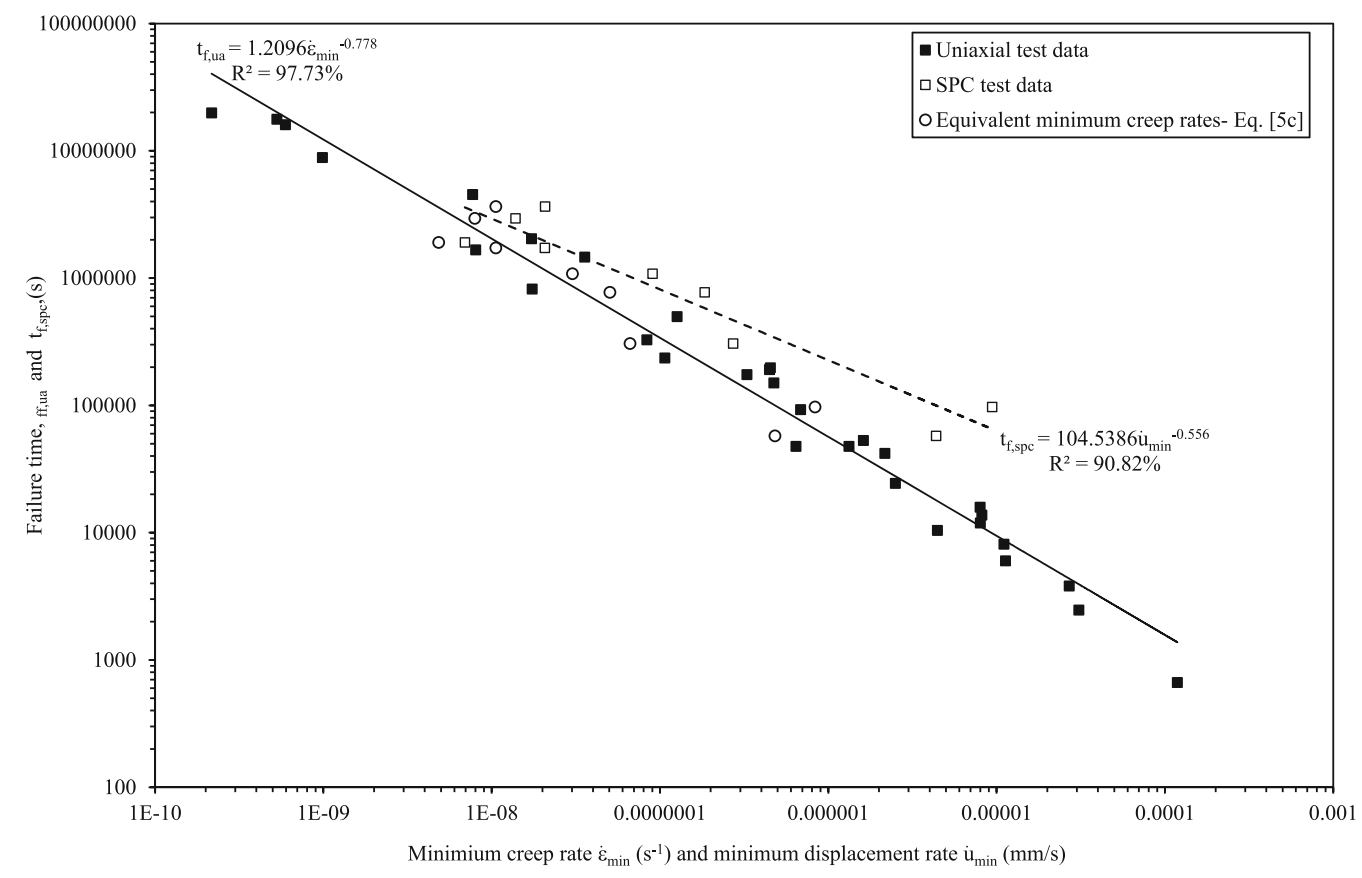

Fig. 4-Monkman-Grant relations for uniaxial and SPC test data.

Figure 6(b) shows a display of the Wilshire equation for the SPC failure times. The horizontal axis shows the temperature-compensated SPC failure times using $g_{2}=$ 359 in Eq. [8b]). The vertical axis is a $\log -\log$ transformation of the normalized force, with the solid line showing the best fit to the data points. The best fit (i.e., largest $R^{2}$ value) is obtained without any discontinuity. This best fit line is given by Eq. [8b] with $g_{0}=-31.236$ and $g_{1}=15.819$. The fit has an $R^{2}$ of just over 93 pct.

The same display is used to represent the Wilshire equation for SPC minimum displacement rates in Figure 6(a). The horizontal axis shows the temperature-compensated minimum displacement rates using $d_{2}$ $=-631$ in Eq. [7b]). The best fit (i.e., largest $R^{2}$ value) is again obtained without any discontinuity. This best fit line is given by Eq. [7b] with $d_{0}=62.64$ and $d_{1}=-$ 25.90. The fit has an $R^{2}$ value of just over 87 pct.

\section{Equivalent Uniaxial Stress for a Given SPC Force}

Figure 7 shows the force-equivalent stress relationships defined by Eqs. [9a] and [9b] over the test conditions present within the uniaxial test data set. Unlike the $k_{\mathrm{sp}}$ method, which implies a linear proportional relationship, this plot reveals quite a complex shape profile between force and the equivalent uniaxial stress. Further, this relationship is clearly temperature dependent.

Figure 8(a) plots the uniaxial stresses that would be equivalent to the nine test forces making up the small punch test data set. The stresses equivalent to these forces are shown on the horizontal axis and were calculated using the $k_{\mathrm{sp}}$ method, i.e., inserting the nine test forces used in the SPC data set into Eq. [1c]. The actual force values generating these equivalent stresses are shown alongside the data points. These equivalent stresses are then plotted against the uniaxial stress interpolated using the Wilshire equation for uniaxial stress-Eq. [10a]. That is, the nine SPC failure times are inserted into Eq. [10a] and then solved for the uniaxial stress required to generate these times at each temperature (using the values for e described above). The square root of the MSPE (RMSPE) associated with the difference between these two stress values is $19.5 \mathrm{pct}$, but the proportion of this that is random in nature is quite low at 11.6 pct. The systematic bias $\left(U^{\mathrm{S}}=88.4\right.$ pct $)$ further reveals itself in the best fit line having a much steeper line than the $45^{\circ}$ line (dashed line) that corresponds to the equivalent and interpolated stresses being equal. At low stresses there is a tendency for the $k_{\mathrm{sp}}$ method to overestimate the interpolated uniaxial stress, while the opposite is true at higher stresses. As a result, on average the equivalent and interpolated stresses are the same (shown by $U^{\mathrm{M}}=0 \mathrm{pct}$ ), but the steep slope of the best fit line (twice as high as the $45 \mathrm{deg}$ line) results in a systematic tendency for the $k_{\mathrm{sp}}$ method to either over or under predict the uniaxial stress at any particular test condition. The value used for $k_{\mathrm{sp}}$ was chosen so as to minimize the above MSPE $\left(k_{\mathrm{sp}}=0.57\right)$.

There are perhaps two interrelated reasons as to why the SPC behavior of Waspaloy, does not follow the $k_{\mathrm{sp}}$ correlation, that has usefully been employed for other creep-resistant alloys: the multiaxial stress state not correlating to uniaxial data and stresses changing between different observed uniaxial creep regimes. First, the creep behavior of Waspaloy has been shown to be dependent on applied condition with two distinct regions corresponding to the stresses above and below $\sigma_{\mathrm{Y}}$ (the yield stress). ${ }^{[20,21]}$ This change in creep behavior is due to differing mechanisms of creep at different applied conditions. Whittaker et al. ${ }^{[21]}$ highlighted the dominance of diffusive climb at stresses below $\sigma_{\mathrm{Y}}$ with 


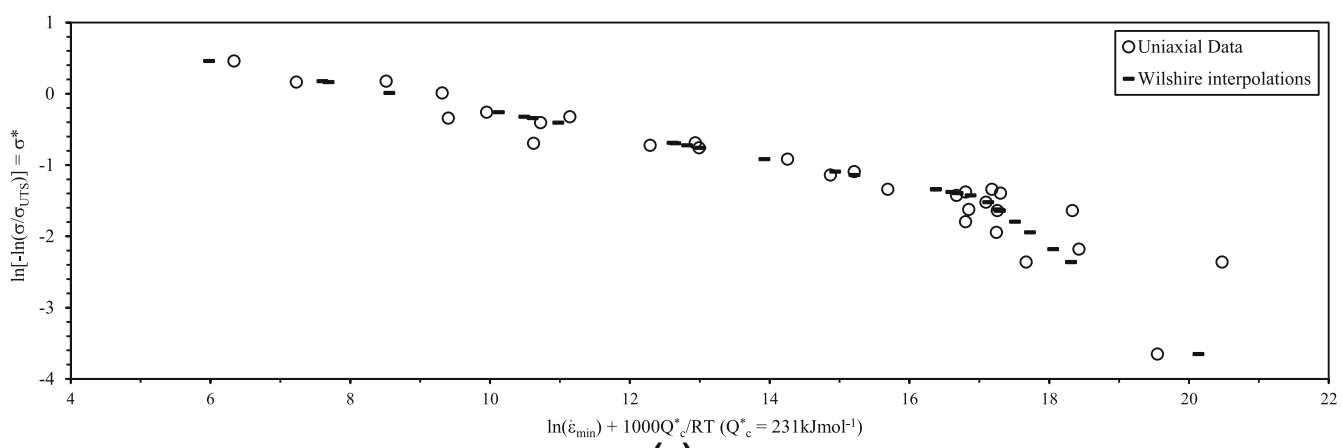

(a)

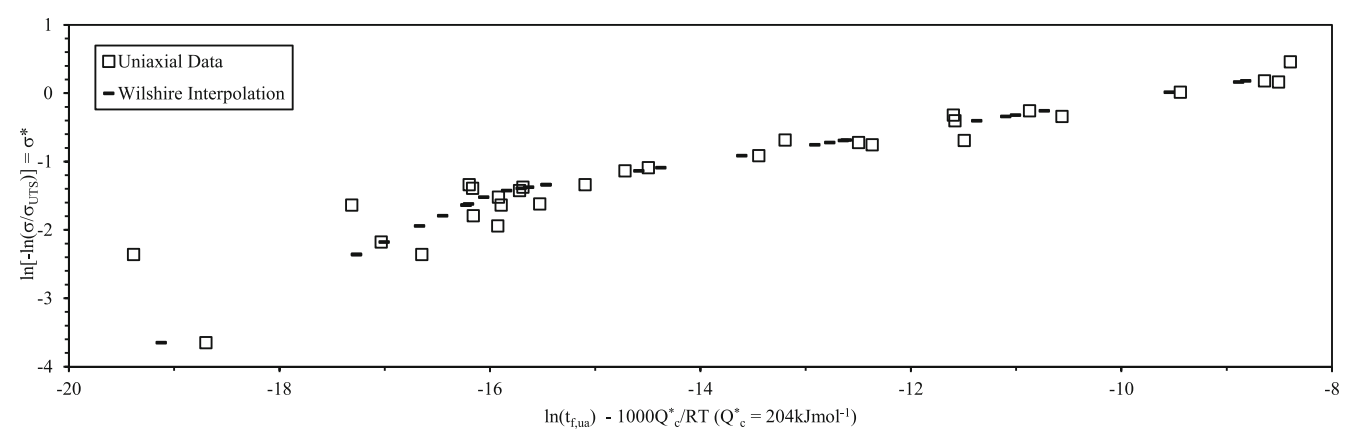

(b)

Fig. 5-The Wilshire representation of uniaxial Waspaloy creep data on (a) minimum creep rates and (b) failure times.

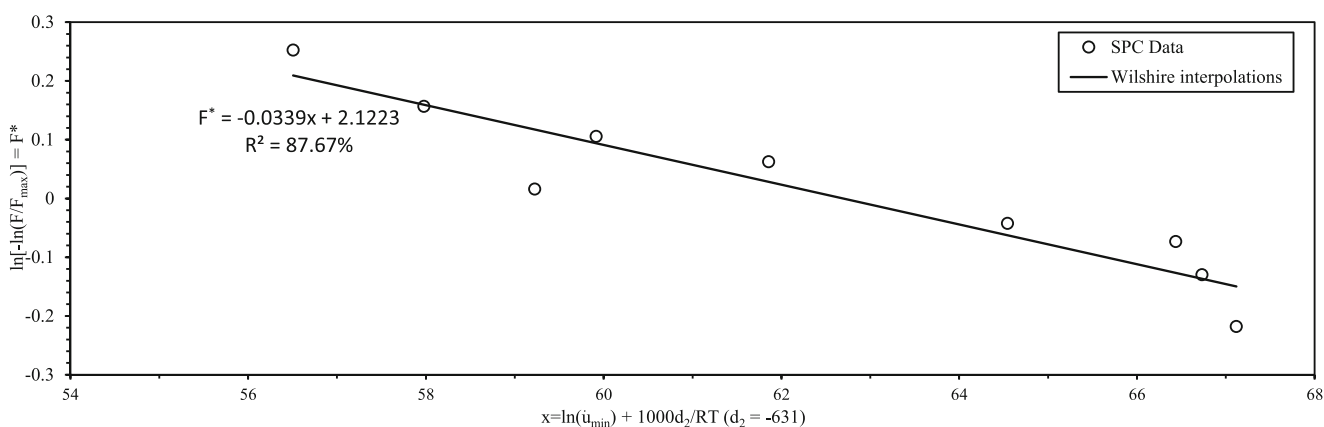

(a)

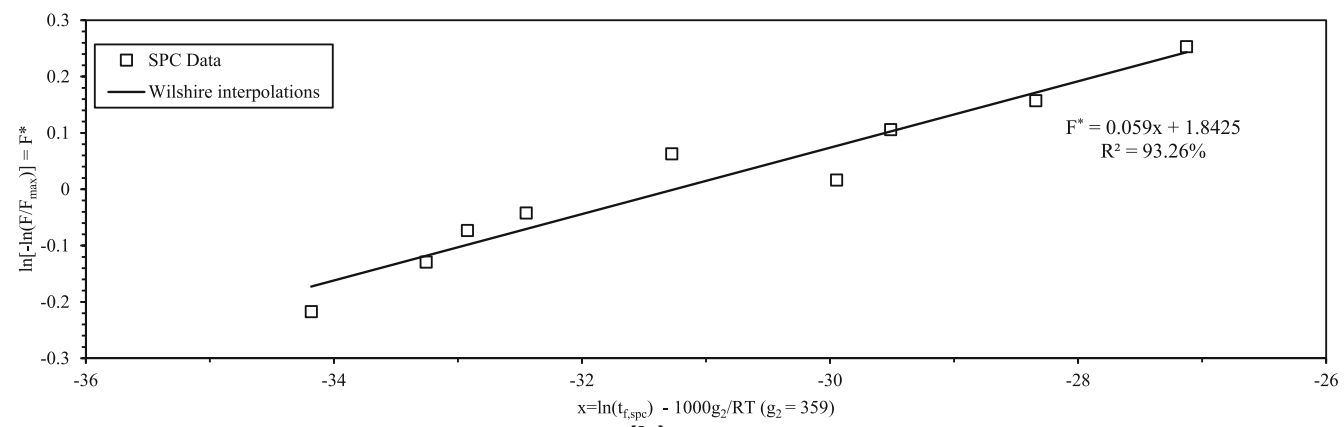

(b)

Fig. 6-The Wilshire representation of SPC Waspaloy creep data on (a) minimum displacement rates and $(b)$ failure times.

dislocation-dislocation interaction in the form of forest hardening limiting creep rates at higher stresses. Birosca et $a l^{[22]}$ showed that geometrically necessary dislocation (GND) densities are higher at the grain boundaries in Waspaloy samples crept below $\sigma_{\mathrm{Y}}$, whereas GND densities were more uniformly spread through grains in samples crept above $\sigma_{\mathrm{Y}}$.

Secondly, the creep behavior of Waspaloy has also been shown to be dependent on the direction of stress, with the maximum principle stress, $\sigma_{1}$ having a strong 


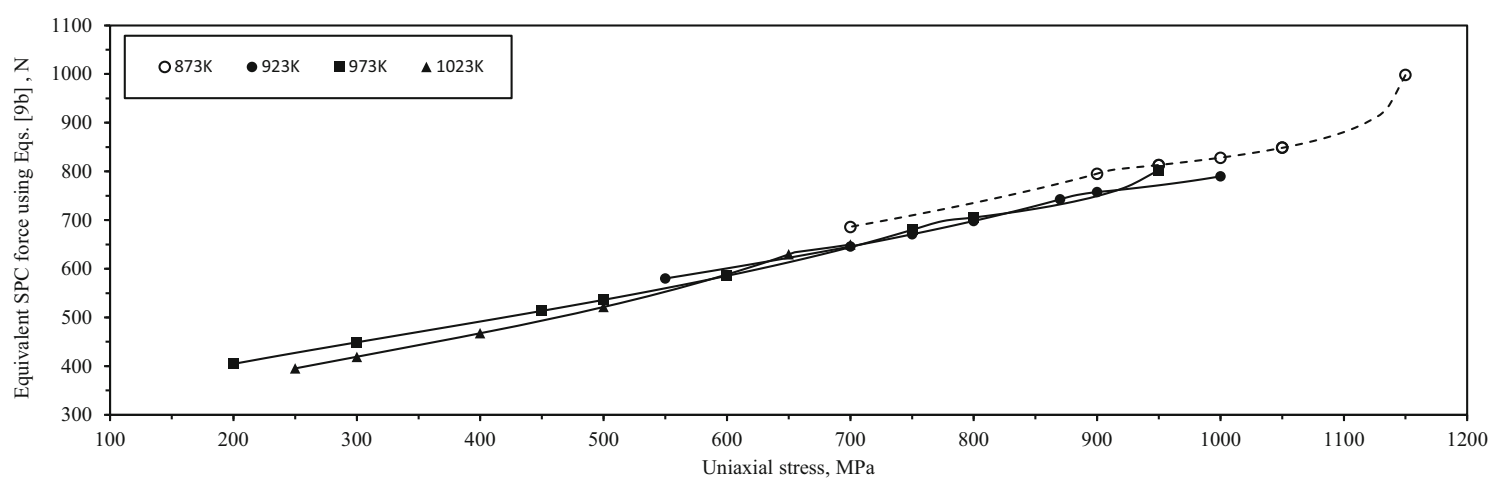

(a)

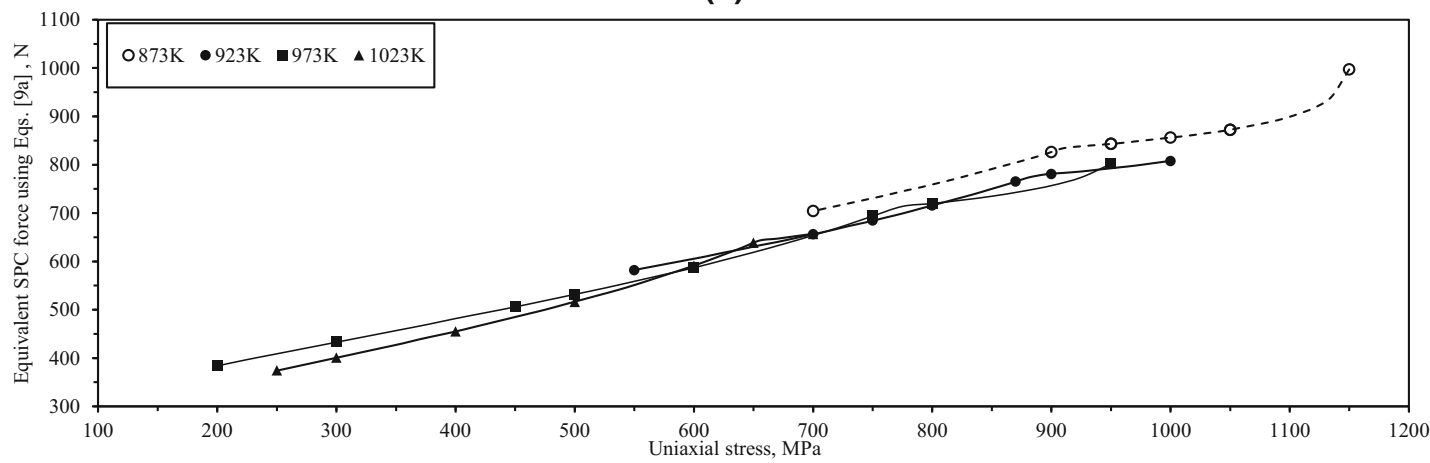

(b)

Fig. 7-Conversion of force to equivalent uniaxial stress over the SPC test condition range using (a) Eq. [9b] and (b) Eq. [9a].

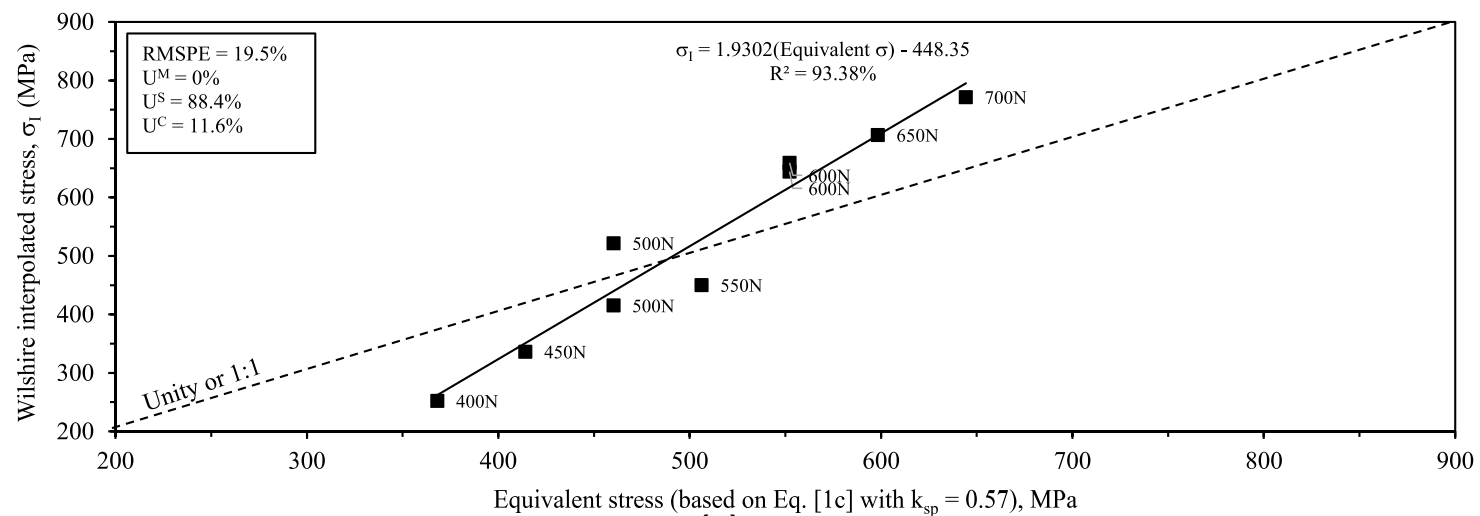

(a)

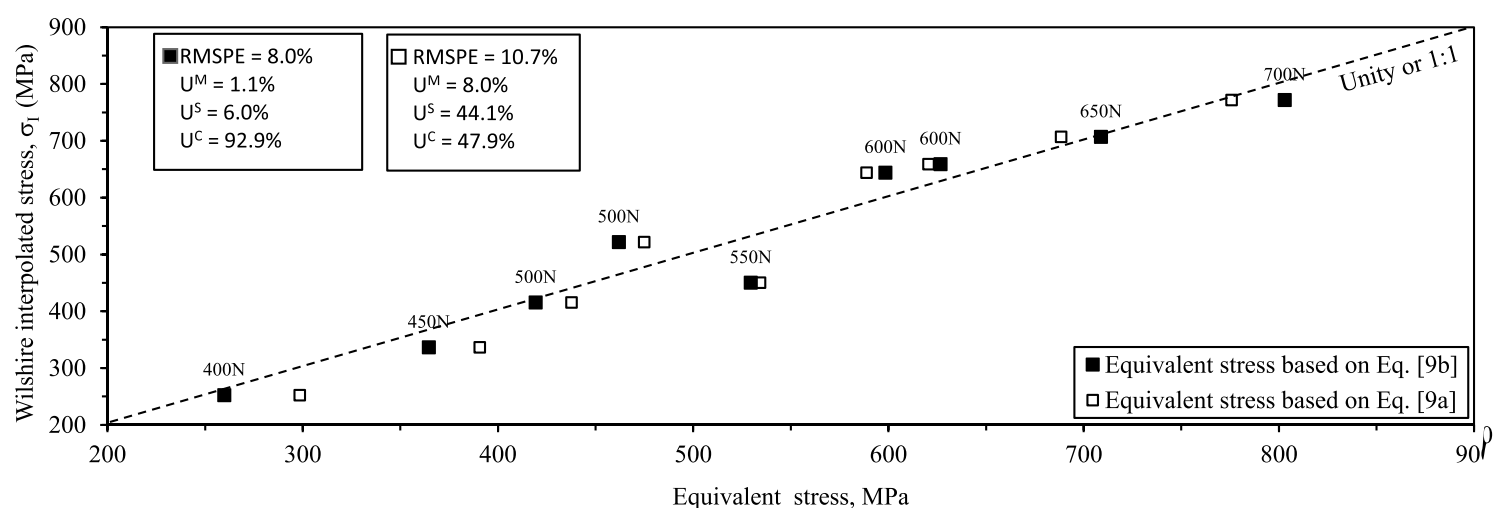

(b)

Fig. 8-Wilshire interpolated uniaxial stress in comparison to the equivalent stress calculated using $(a)$ the $k_{\mathrm{sp}}$ method and (b) the approaches given by Eqs. [9a] and [9b]. 
influence on tertiary creep and rupture. ${ }^{[23]}$ This dependence on the stress state will have an influence on the SPC behavior of Waspaloy, since the stress state is non-uniform and evolves during the test. Initially, the maximum stress is compressive, immediately adjacent to the punch, before becoming more tensile across the width of the disc. A combination of high radial and hoop stresses results in a peak maximum principle stress on the underside of the disc, whereas the von-Mises stress is distributed across the thickness of the disc, near the point of contact with the punch. ${ }^{[23]}$ Harrison et al. ${ }^{[24]}$ showed that creep rates in Waspaloy during stress and temperature change experiments can be higher than expected. It was proposed that this is due to the breakdown of stable dislocation structures as applied conditions change.

The Wilshire equations allow for changing mechanisms of deformation by having break points (as seen in Figure 5) and so offer the possibility of being able to more accurately calculate equivalent stresses and forces. This is born out in Figure 8(b), that shows the same uniaxial stresses as in Figure 8(a) (interpolated using the Wilshire equation for uniaxial stress-Eq. [10a]), but this time they are cross plotted against the stresses equivalent to these forces as calculated using either Eq. [9a] (corresponding to the open square symbols) or Eq. [9b] (corresponding to the filled square symbols). Take first the equivalent stresses obtained using Eq. [9b]. The RMSPE associated with the difference between these two stress values is 8.0 pct and the proportion of this that is random in nature is very high at 92.9 pct. The remaining systematic bias is mainly due to $U^{\mathrm{S}}=6.0$ pct, so that on average the equivalent and interpolated stresses are almost same (shown by $U^{\mathrm{M}}=1.1 \mathrm{pct}$ ). Next consider the equivalent stresses obtained using Eq. [9a]. The RMSPE associated with the difference between these two stress values is a little higher at 10.7 pct and the proportion of this that is random in nature is lower at 47.9 pct. The remaining systematic bias is mainly due to $U^{\mathrm{S}}=44.1 \mathrm{pct}$. This approach, that is based on equating minimum creep and deformation rates rather than uniaxial and SPC failure times, has characteristics of accuracy somewhere in between the other two approaches shown in Figure 8.

\section{Equivalent SPC Force for a Given Uniaxial Stress}

The analysis in subsection $\mathrm{C}$ above was based on only nine data points. To further assess the suitability of various conversion approaches, the 31 stresses making up the uniaxial data set are converted to equivalent SPC forces using the different conversion methods. The results are shown in Figure 9. Figure 9(a) plots the SPC forces equivalent to the 31 test stresses making up the uniaxial data set. The forces equivalent to these uniaxial stresses are shown on the horizontal axis and were calculated using the $k_{\mathrm{sp}}$ method, i.e., inserting the 31 test stresses used in the uniaxial data set into Eq. [1b]. Some of the actual stress values generating these equivalent forces are shown alongside the data points. These equivalent forces are then plotted against the SPC forces interpolated using the Wilshire equation for force-Eq. [10b]. That is, the 31 uniaxial failure times are inserted into Eq. [10b] and then solved for the SPC force required to generate these times at each temperature (using values for g shown above). The RMSPE associated with the difference between these two forces values is 22.6 pct, but the proportion of this that is random in nature is quite low at just over 11.9 pct. The systematic bias ( $U^{\mathrm{S}}=88 \mathrm{pct}$ ) further reveals itself in the best fit line having a much flatter line than the $45 \mathrm{deg}$ line (dashed line) that corresponds to the equivalent and interpolated forces being equal. At low forces there is a tendency for the $k_{\mathrm{sp}}$ method to underestimate the interpolated SPC force, while the opposite is true at higher forces. As a result, on average the equivalent and interpolated forces are the same (shown by $U^{\mathrm{M}}=0.1$ pct), but the shallow slope of the best fit line (half as high as the 45 deg line) results in a systematic tendency for the $k_{\mathrm{sp}}$ method to either over or under predict the SPC force at any particular test condition. Again the value for $k_{\mathrm{sp}}(0.52)$ was chosen to minimize the above MSE. These results are very similar to when working with equivalent stresses in Figure 8(a).

Figure 9(b) shows the same SPC forces as in Figure 9(a) (interpolated using the Wilshire equation for force-Eq. [10b]), but this time these are cross plotted against the forces equivalent to the 31 uniaxial stresses as calculated using either Eq. [9a] (corresponding to the open square symbols) or Eq. [9b] (corresponding to the filled square symbols). Take first the equivalent forces obtained using Eq. [9b]. The RMSPE associated with the difference between these two stress values is 3.6 pct and the proportion of this that is random in nature is very high at 95.4 pet. The remaining systematic bias is mainly due to $U^{\mathrm{S}}=3.5 \mathrm{pct}$, so that on average the equivalent and interpolated forces are almost the same (shown by $\left.U^{\mathrm{M}}=1.1 \mathrm{pct}\right)$. Next consider the equivalent forces obtained using Eq. [9a]. The RMSPE associated with the difference between these two force values is a little higher at 4.8 pct and the proportion of this that is random in nature is lower at 56.1 pct. The remaining systematic bias is mainly due to $U^{\mathrm{S}}=38.8$ pct. Again these results are consistent with those shown in Figure 8.

\section{E. Accuracy of Conversion Methods Based of Predicted Failure Times}

The failure times shown along the horizontal axis of Figure 10(a) were computed by first converting the 31 uniaxial stresses into equivalent forces using Eq. [1c], i.e., using the $k_{\mathrm{sp}}$ method with $k_{\mathrm{sp}}=0.52$. These forces were then inserted into Eq. [8b] to predict a failure time for an equivalent SPC test. If the equivalence calculation is accurate, these predicted failure times should equal the actual uniaxial failure times - which are therefore shown on the vertical axis of Figure 10(a). But as can be seen from this figure, the best fit line through all the data points is much flatter that the $45 \mathrm{deg}$ line. It is therefore not surprising to note that the RMPSE is as high as 367 pct, with only 29.5 pct of this error being random in nature. 


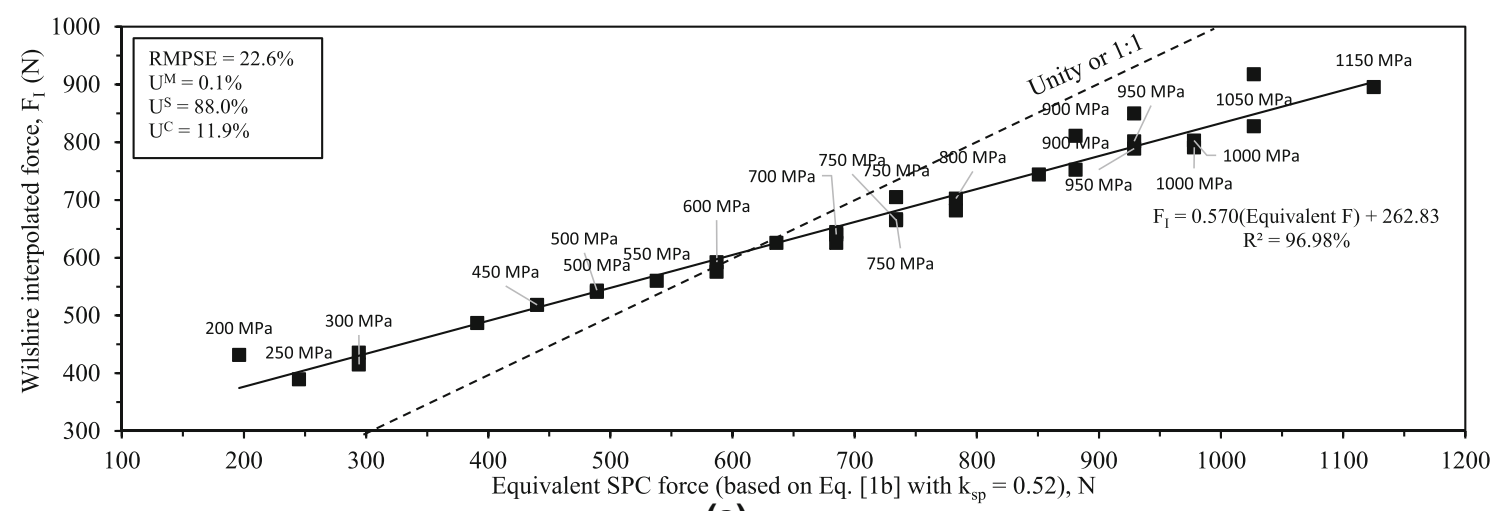

(a)

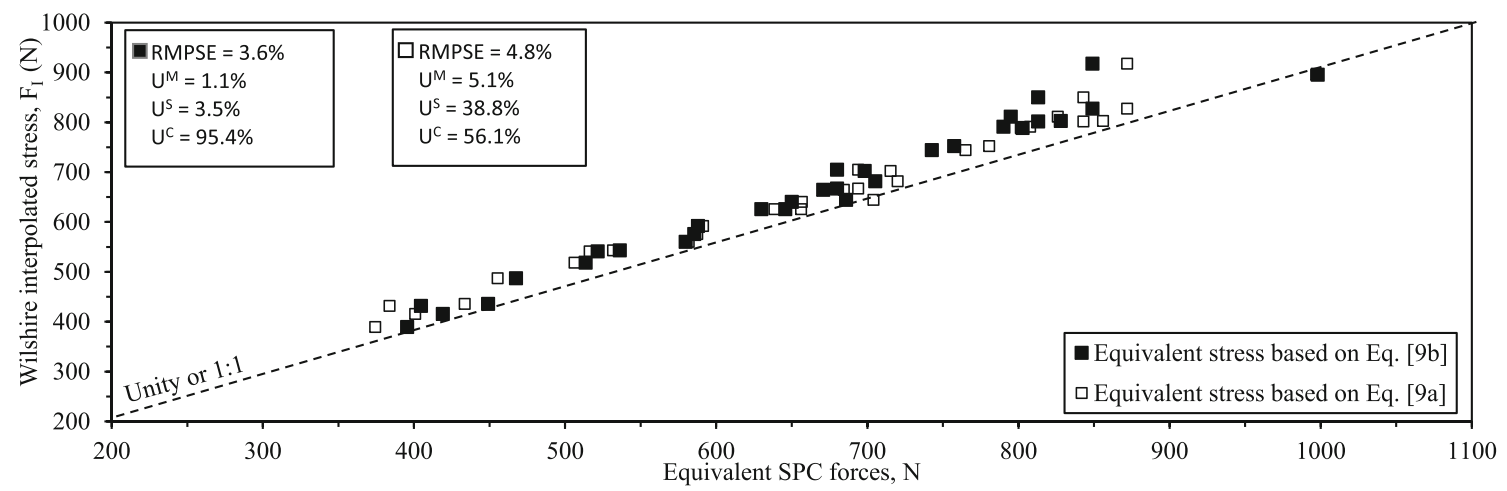

(b)

Fig. 9-Wilshire interpolated SPC force in comparison to the equivalent force calculated using $(a)$ the $k_{\mathrm{sp}}$ method and (b) the approaches given by Eqs. [9a] and [9b].

The failure times shown along the horizontal axis of Figure 10(b) were computed by first converting the 31 uniaxial stresses into equivalent forces using Eq. [9b]. These forces were then inserted into Eq. [8b] to predict a failure time for an equivalent SPC test. If this equivalence calculation is accurate, the predicted failure times should equal the actual uniaxial failure times - which are therefore shown on the vertical axis of Figure 10(b). As can be seen from this figure the best fit line through all the data points are very similar to the $45^{\circ}$ linehaving an exponent close to unity-0.92. It is therefore not surprising to note that the RMPSE is much lower compared to when using the $k_{\mathrm{sp}}$ method-86.0 pctwith much of this error being random in nature-97.5 pct.

The failure times shown along the horizontal axis of Figure 10(c) were computed by first converting the 31 uniaxial stresses not equivalent forces using Eq. [9a]. These forces were then inserted into Eq. [8b] to predict a failure time for an equivalent SPC test. If this equivalence calculation is accurate, the predicted failure times should equal the actual uniaxial failure times-which are therefore shown on the vertical axis of Figure 10(c). As can be seen from this figure, the best fit line through all the data points is very similar to the $45 \mathrm{deg}$ line-having an exponent close to unity-0.86. It is therefore not surprising to note that the RMPSE is much lower compared to when using the $k_{\mathrm{sp}}$ method-99.6 pet-but a little higher than that shown in Figure 10(b). Around 80 pet of this error is random in nature.

\section{CONCLUSION}

The main aim of this paper was to accurately convert small punch loads into equivalent uniaxial stresses. This paper introduced a number of alternative approach to the widely used constant stress to force ratio methods. These alternatives included the use of the MonkmanGrant relation to convert minimum displacement rates into equivalent minimum uniaxial creep rates and the application of the Wilshire equations to SPC test data using maximum forces in place of UTS. These alternative techniques were then compared to the $k_{\mathrm{sp}}$ method through statistical evaluations of predictions and experimental data. The following conclusions could be drawn:

- The $k_{\mathrm{sp}}$ method was not appropriate for correlating uniaxial and SPC data for this composition of Waspaloy, with the method producing large prediction errors. Further, the vast majority of these predictions errors were non-random in nature.

- The Monkman-Grant grant relationships for the small punch and uniaxial creep data were shown to have similar slopes will dissimilar degrees of fit. 


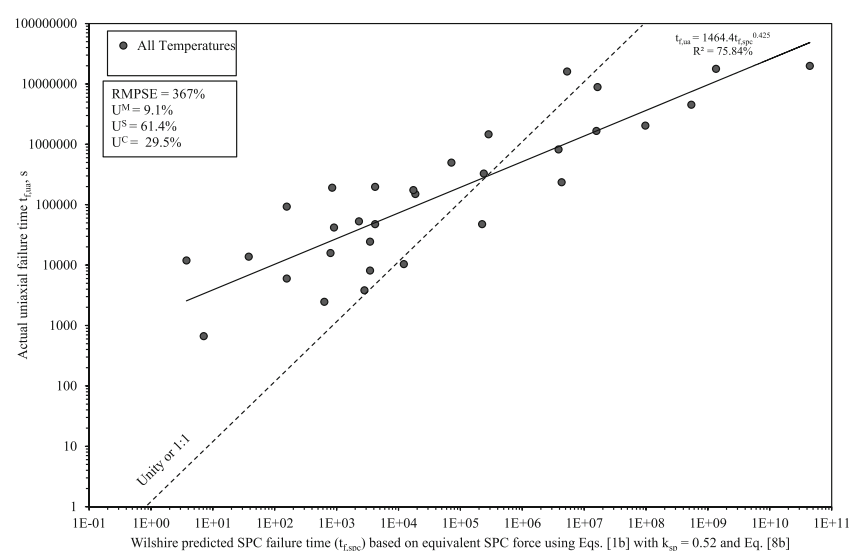

(a)

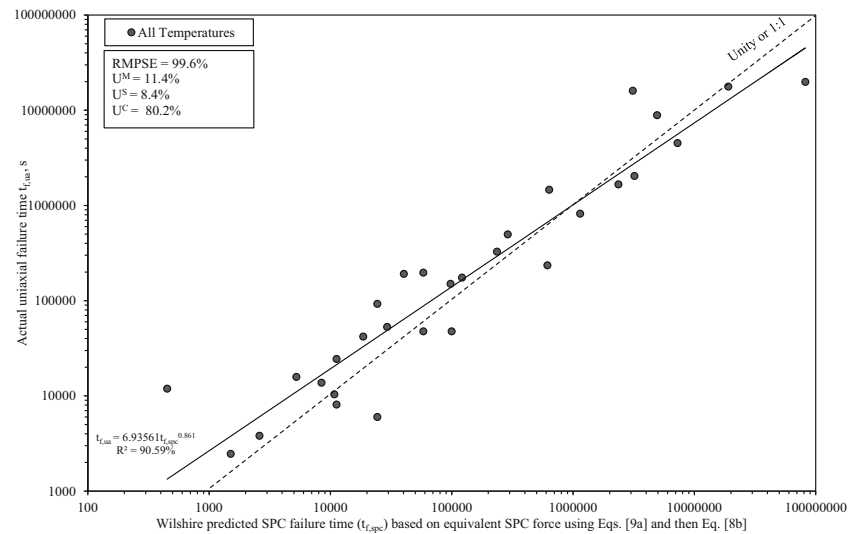

(c)

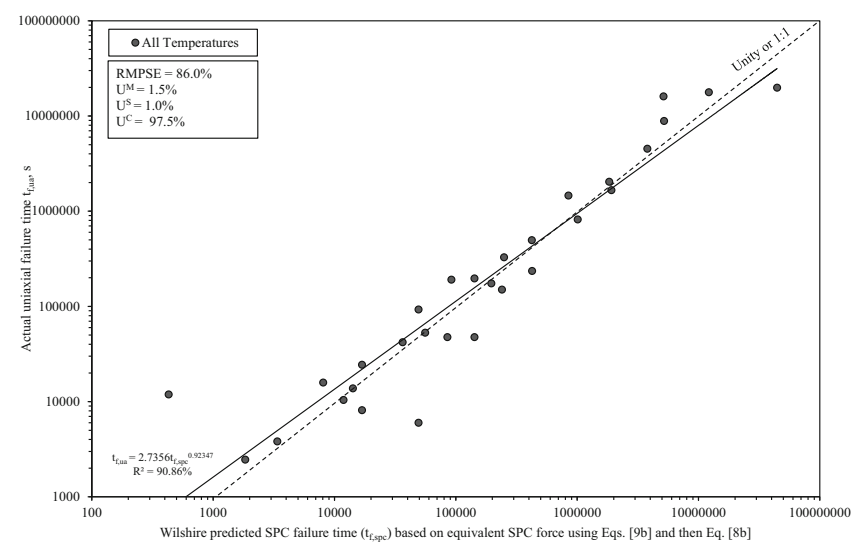

(b)

Fig. 10 - (a) Predicting uniaxial failure times from SPC tests when using the $k_{\mathrm{sp}}$ method to convert stresses to equivalent forces. $(b)$ Predicting uniaxial failure times from SPC tests when using Eq. [9b] to convert stresses to equivalent forces. (c) Predicting uniaxial failure times from SPC tests when using Eq. [9a] to convert stresses to equivalent forces.

Consequently, a shifting of the SPC MonkmanGrant relation would be only approximate for the conversion of minimum displacement rates to equivalent uniaxial minimum creep rates.

- When these predicted minimum creep rates were combined with the Wilshire equations for minimum creep and displacement rates, SPC loads were converted to equivalent uniaxial stresses with degrees of accuracy that were much better than those obtained using the $k_{\mathrm{sp}}$ method. Further, and unlike when using the $k_{\mathrm{sp}}$ approach, these prediction errors were predominantly random in nature. The approach was also better able to predict times to failure.

- The most accurate approach to converting force to stress was, however, obtained by modeling failure times under both types of test using a Wilshire type equation.

\section{ACKNOWLEDGMENTS}

The authors would like to thank Rolls-Royce for their financial support and technical involvement in this research, and in supplying some of the experimental data and test material used within this paper.

\section{OPEN ACCESS}

This article is licensed under a Creative Commons Attribution 4.0 International License, which permits use, sharing, adaptation, distribution and reproduction in any medium or format, as long as you give appropriate credit to the original author(s) and the source, provide a link to the Creative Commons licence, and indicate if changes were made. The images or other third party material in this article are included in the article's Creative Commons licence, unless indicated 
otherwise in a credit line to the material. If material is not included in the article's Creative Commons licence and your intended use is not permitted by statutory regulation or exceeds the permitted use, you will need to obtain permission directly from the copyright holder. To view a copy of this licence, visit http://creat ivecommons.org/licenses/by/4.0/.

\section{REFERENCES}

1. R.J. Lancaster, S.P. Jeffs: Small Punch Creep. Creep [Internet]. 2018; Available from: http://www.intechopen.com/books/creep/s mall-punch-creep.

2. C. Wen, T. Xu, and K. Guan: Correlation factor study of small punch creep test and its life predictionMaterials, 2016, vol. 9 (10), p. 796.

3. K. Matocha and R. Hurst: Small punch testing-the transition from a code of practice to a European testing standardKey Eng. Mater., 2017, vol. 3 (22), p. 734.

4. J. Chakrabarty: A theory of stretch forming over hemispherical punch headsInt. J. Mech. Sci., 1970, vol. 12 (4), pp. 315-25.

5. S.P. Jeffs, R.J. Lancaster, and T.E. Garcia: Creep lifing methodologies applied to a single crystal superalloy by use of small scale test techniquesMater. Sci. Eng. A, 2015, vol. 636, pp. 529-35.

6. K. Milička and F. Dobeš: Small punch testing of P91 steelInt. J. Pressure Vessel Piping, 2006, vol. 83, pp. 625-34.

7. R.J. Lancaster, W.J. Harrison, and G. Norton: An analysis of small punch creep behaviour in the $\gamma$ titanium aluminide Ti-45Al-2Mn-2NbMater. Sci. Eng. A, 2015, vol. 626, pp. 263-74.

8. S. Holmström, Y. Li, P. Dymacek, E. Vacchieri, S.P. Jeffs, R.J. Lancaster, D. Omacht, Z. Kubon, E. Anelli, J. Rantala, A. Tonti, and S. Komaz: Creep strength and minimum strain rate estimation from Small Punch creep testsMater. Sci. Eng. A, 2018, vol. 731, pp. 161-72.

9. M. Evans and D. Wang: The small punch creep test: some results from a numerical modelJ. Mater. Sci., 2008, vol. 43 (6), pp. 1825-35.

10. G. Sundararajan: The Monkman-Grant relationshipMater. Sci. Eng. A, 1989, vol. 112, pp. 205-14.

11. F. Dobeš and K. Milička: On the Monkman-Grant relation for small punch test dataMater. Sci. Eng. A, 2002, vol. 336 (1-2), pp. $245-48$.
12. B. Wilshire and A.J. Battenbough: Creep and creep fracture of polycrystalline copperMater. Sci. Eng. A, 2007, vol. 443 (1-2), pp. $156-66$.

13. R.W. Evans and B. Wilshire: Introduction to Creep, 1st ed., Institute of Materials, London, 1993, pp. 23-28.

14. B. Wilshire and P.J. Scharning: Theoretical and practical approaches to creep of WaspaloyMater. Sci. Technol., 2009, vol. 25 (2), pp. $242-48$.

15. R.W. Evans: A constitutive model for the high-temperature creep of particle-hardened alloys based on the $\theta$ projection methodProc. R. Soc. Lond. A, 2000, vol. 456, pp. 835-68.

16. CEN. Part A: A Code of Practice for Small Punch Creep Testing. Small Punch Test Method Met Mater. 2006.

17. S. H. Song, J. Wu, X.J. Wei, D. Kumar, S.J. Liu and L.Q. Weng: Creep property evaluation of a $2.25 \mathrm{Cr}-1 \mathrm{Mo}$ low alloy steel, Mater. Sci. Eng. A, 2010, 527(9):2398-403.

18. M. Evans: The importance of creep strain in linking together the Wilshire equations for minimum creep rates and times to various strains (including the rupture strain): An illustration using $1 \mathrm{CrMoV}$ rotor steelJ. Mater. Sci., 2014, vol. 49 (1), pp. 329-39.

19. H. Theil: Applied Economic Forecasts, North Holland, Amsterdam, 1966.

20. M. Whittaker, W. Harrison, C. Deen, C. Rae, S. Williams: Creep deformation by dislocation movement in Waspaloy, Materials (Basel). 10 2017. https://doi.org/10.3390/ma10010061.

21. M. Evans and T. Williams: Assessing the capability of the Wilshire equations in predicting uniaxial creep curves : an application to WaspaloyInt. J. Pressure Vessel. Piping, 2019, vol. 172, pp. 153-65, https://doi.org/10.1016/j.ijpvp.2019.03.032.

22. S. Birosca, G. Liu, R. Ding, J. Jiang, T. Simm, C. Deen, and M. Whittaker: The dislocation behavior and GND development in a nickel based superalloy during creepInt. J. Plast, 2019, vol. 118, pp. 252-68, https://doi.org/10.1016/j.ijplas.2019.02.015.

23. R.J. Lancaster, W.J. Harrison, and G. Norton: An analysis of small punch creep behavior in the $\gamma$ titanium aluminide Ti-45Al-2Mn-2NbMater. Sci. Eng. A, 2015, vol. 626, pp. 263-74, https://doi.org/10.1016/j.msea.2014.12.045.

24. W.J. Harrison, M.T. Whittaker, and C. Deen: Creep behavior of Waspaloy under non-constant stress and temperatureMater. Res. Innov., 2013, vol. 17, pp. 323-26.

Publisher's Note Springer Nature remains neutral with regard to jurisdictional claims in published maps and institutional affiliations. 